Giornale di Tecniche

\title{
Dialisi peritoneale: perché dialisi marginale?
}

\author{
Sergio Sisca \\ Ospedale Santa Maria Annunziata \\ ASL 10, Firenze
}

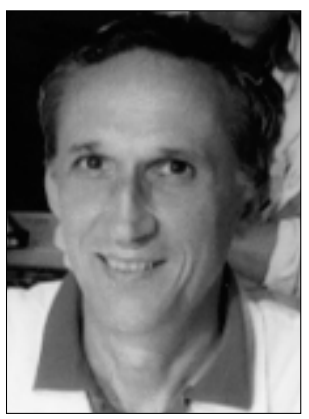

P ispondo volentieri ai quesiti posti da Marco Lombardi sulla dialisi peritoneale perché mi sembrano di grande importanza e di enorme attualità. Secondo il mio parere sono tre $\mathrm{i}$ motivi che relegano la dialisi peritoneale a un posto marginale (in Italia alla fine del 1998 soltanto il $12 \%$ era trattato con questa metodica): scarsa convinzione dei medici, disinteresse delle strutture sanitarie private, disinteresse delle ditte biomediche.

\section{Scarsa convinzione dei medici}

Al momento di inserire un paziente in un programma di terapia sostitutiva della funzione renale, il nefrologo si pone il problema (Fig. 1) se utilizzare l'emodialisi (ED) o la dialisi peritoneale (DP). Tale scelta è condizionata da una serie di motivazioni: convinzioni del nefrologo, disponibilità strutturali, scelta del paziente e sue condizioni cliniche.

In tale visione "dicotomica" tra le due procedure, numerosi ricercatori hanno tentato di dimostrare la supe- riorità di una metodica rispetto all'altra nel breve e lungo termine senza però giungere a conclusioni definitive. Proprio per questo, purtroppo, non si capisce bene come nonostante i vantaggi della dialisi peritoneale, riconosciuti dalla letteratura internazionale, alcuni nefrologi considerano ancora la peritoneale una dialisi di seconda scelta dove poter indirizzare i pazienti più anziani, diabetici, cardiopatici, con gravi patologie associate, in altre parole quelli che per controindicazioni cliniche e/o sociali ai trattamenti extracorporei sono destinati a morte sicura. Questa scelta ha determinato delle curve di sopravvivenza estre- mamente svantaggiose per la dialisi peritoneale che hanno creato nel passato un comprensibile scetticismo nella cultura della maggior parte dei nefrologi.

Oggi una più calibrata allocazione dei pazienti, il miglioramento tecnologico della peritoneale, una più appropriata metodologia statistica che prevede un'accurata analisi dei dati con stratificazione dei pazienti secondo differenti criteri di selezione, fattori di rischio, età anagrafica ecc., hanno dimostrato una sopravvivenza dei dializzati pressoché uguale sia in ED che in DP. Non è, pertanto, più giustificato un atteggiamento ostativo

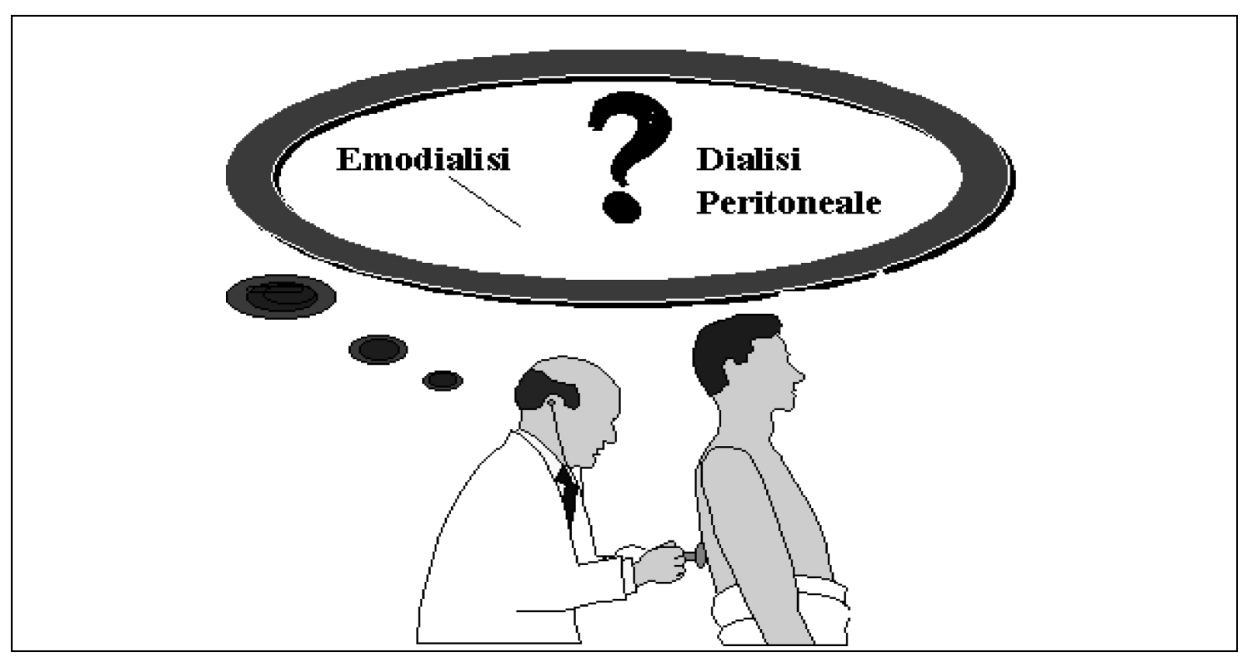

Fig. 1 - Il dilemma di sempre. 


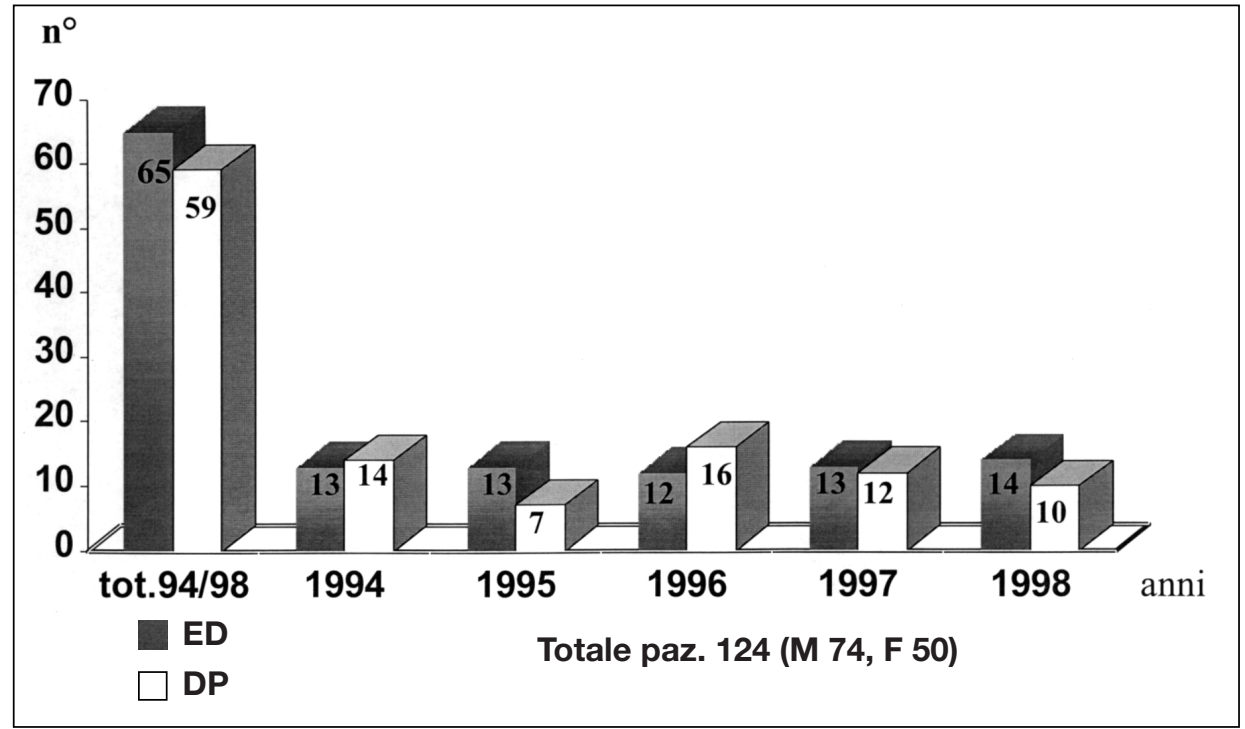

Fig. 2 - Nuovi ingressi 1994/98.

nei confronti di una tecnica dialitica ormai consolidata.

La domanda che vorrei porre a questi nefrologi, poco entusiasti della peritoneale, è questa:

Chi deve scegliere per un paziente affetto da insufficienza renale cronica un trattamento che ne condizionerà completamente la vita lavorativa, sociale, familiare, psicologica (essere dipendente da una macchina e dai ritmi del trattamento) e che ne limiterà le relazioni interpersonali, il tempo libero, l'assunzione di cibi liquidi o solidi? Troppo spesso è il medico che si assume questa responsabilità secondo le proprie convinzioni, e questo non è al giorno d'oggi giustificabile.

La scelta secondo me dovrebbe essere un diritto del paziente dopo averne discusso con il nefrologo. In considerazione del fatto che oggi si possono offrire varie soluzioni in grado di rispondere alle singole esigenze di ciascuno, che tutte le modalità di dialisi sono efficienti ed efficaci, ciascuna naturalmente con i propri vantaggi e svantaggi, che esistono poche controindicazioni per entrambe le metodiche, sarebbe opportuno adeguare la dialisi alle specifiche esigenze del paziente e non il paziente alla dialisi. Secondo il Comitato Nazionale di Bioetica: "oggi si ravvisa la necessità del passaggio dal rapporto paternalistico in cui il medico decideva in scienza e coscienza per il bene del paziente a quello che prevede una maggiore partecipazione del paziente alle decisioni che lo riguardano". $\mathrm{Ci}$ si sta dunque avviando a un passaggio dal "paziente passivo" al "paziente razionale" (Tab. I). Per giungere alla scelta del trattamento migliore però il paziente deve disporre di informazioni esaurienti su tutte le opzioni disponibili. Ma come si possono dare informazioni complete nei Centri di nefrologia dove la dialisi peritoneale è assolutamente "vietata"?

In alcuni Centri i fattori determinanti nella scelta sono costituiti dal costo del trattamento, che dovrebbe invece essere tenuto in scarsa considerazione, da mancanza di posti tecnici di

\section{TABELLA I - DAL "PAZIENTE PASSIVO" AL "PAZIENTE RAZIONALE"}

Passato

Non è informato

Non ha alcuna educazione sanitaria

Non richiede trattamenti specifici

Non rispetta le prescrizioni del medico

È insoddisfatto dell'assistenza, ma non '̇̀ insoddisfatto dell'assistenza e prende prende iniziative autonome
Richiede trattamenti specifici

Rispetta le prescrizioni del medico solo in caso di sintomi o patologie più gravi

\section{Presente}

È informato, ma prevalentemente dal medico o da materiali divulgativi

Riceve scarsissima educazione sanitaria iniziative autonome per garantirsi una migliore qualità delle cure

\section{Futuro}

È informato in materia sanitaria e riguarda alla sua salute

Ha una buona educazione sanitaria e richiede informazione di più alto livello qualitativo

Richiede trattamenti specifici e rivendica il suo diritto di scegliere

Rispetta le prescrizioni del medico

È insoddisfatto dell'assistenza perché ha un ruolo attivo nelle decisioni che lo riguardano 
emodialisi, e dal sempre presente alibi della carenza di personale infermieristico, che impedirebbe lo "storno" anche di uno solo di essi per la peritoneale.

Quest'ultimo problema è presto sfatato dal fatto che la dialisi peritoneale rappresenta un notevole risparmio di personale, infatti il rapporto comparativo infermiere/paziente è di $1 / 3.3$ in EDO e di $1 / 13$ in DP.

Secondo i dati ANED agli inizi degli anni '90 in Toscana venivano trattati in dialisi peritoneale il 3\% dei pazienti, contro una media nazionale dell'8\%; inoltre soltanto 4 Centri su $27(15 \%)$ effettuavano questa metodica. Arrivati in Toscana alla fine degli anni '80 dopo l'esperienza di Reggio Calabria, nel 1991 già trattavamo in peritoneale il $10 \%$ dei nostri pazienti e dal 1995 abbiamo raggiunto una prevalenza del $31 \%$, che è rimasta costante negli anni successivi.

Abbiamo potuto ottenere questo obiettivo perché, ritenendo che l'emodialisi e la dialisi peritoneale debbano essere utilizzate in maniera integrata e sequenziale, siamo riusciti a programmare i nuovi ingressi dal 1994 al 1998 in maniera molto equilibrata (Fig. 2). La peritoneale come prima forma di trattamento, secondo noi, può costituire una sorta di camera di compensazione in considerazione della carenza dei posti tecnici di $\mathrm{ED}$, per cui l'ingresso in dialisi extracorporea potrà essere meglio programmato. Noi siamo stati sempre pronti a cambiare metodica non appena questa si è dimostrata controindicata per motivi clinici e/o sociali.

Non è inoltre da sottovalutare il fatto che considerare la DP come forma elettiva di primo trattamento per i pazienti più giovani, con diuresi residua e con buone condizioni cliniche, offre il vantaggio che tali pazienti possano venir trapiantati in un arco ragionevole di tempo, senza aver mai "intasato" i già stracolmi letti tecnici di emodialisi ospedaliera, ad assistenza limitata o decentrata.

Per restare alla nostra esperienza, se non avessimo adottato tale politica, avremmo dovuto certamente instaurare un terzo turno di dialisi ospedaliero o prevedere la realizzazione di

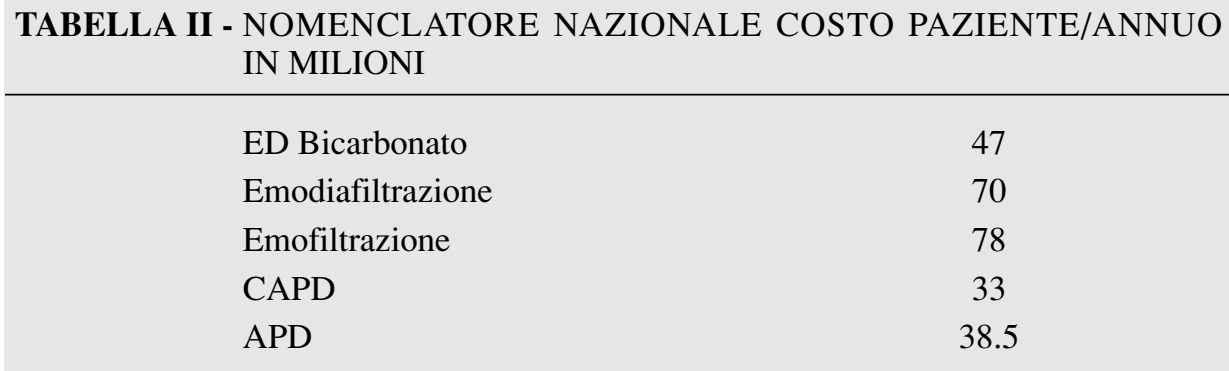

un nuovo Centro dialisi ad assistenza limitata, con tutti i costi conseguenti. Con il nostro esempio e quello dei centri di Arezzo, Siena, Careggi la dialisi peritoneale in questi ultimi anni si è sviluppata anche in Toscana tanto che 21 Centri su 28 (75\%) nel 1999 avevano attivato questa metodica e i pazienti trattatati erano 1'11\% del totale, esattamente in linea con la media nazionale.

\section{Disinteresse delle strutture sanitarie private}

Se era incomprensibile in passato la riluttanza dei centri toscani a trattare i pazienti con dialisi peritoneale, ben chiara è la scarsa penetrazione di questa tecnica in quelle regioni dove i centri privati superano quelli pubblici. In queste regioni, infatti, le strutture private non ritengono remunerativo il rimborso del costo della DP ai sensi del nomenclatore nazionale (Tab. II) e lo dimostrano le statistiche relative al Registro nazionale SIN 1998: la DP è utilizzata nel $1.2 \%$ in Sicilia, e nel 3.6\% nel Lazio; della Campania non si conoscono i dati, anche se si avvicinano presumibilmente alle altre due regioni. Queste percentuali sono del tutto sovrastimate dal fatto che la maggior parte dei Centri privati, che non hanno nel loro programma la DP, non inviano i loro dati ai vari Registri regionali.

\section{Disinteresse delle ditte biomediche}

Il terzo motivo della marginalità della DP è dovuto alla totale indifferenza che le maggiori industrie specia- lizzate in prodotti dialitici hanno nei confronti della CAPD. Eppure dalla storia della DP risulta che questa sia nata prima della ED.

Le prime esperienze sul lavaggio peritoneale risalgono, nel 1877 , a Wegner.

Egli aveva notato che l'aggiunta al liquido di infusione di zucchero o glicerina provocava un aumento della quantità del liquido drenato. Starling nel 1894 studiò i processi di scambio notando l'assorbimento dal peritoneo di blu di metilene e indaco carminio. Putnam nel 1922 si convinse che il peritoneo si poteva comportare come membrana dializzante e l'anno successivo Ganter usò questa metodica prima per dializzare cani uremici e successivamente una donna allungandole la vita per un breve periodo di tempo. Negli anni '40 e '50 la peritoneale era utilizzata esclusivamente nel trattamento dell'insufficienza renale acuta. Negli anni '60 la scoperta degli accessi vascolari (shunt arterovenosi) e l'interesse dell'industria farmaceutica per le apparecchiature di emodialisi e i materiali di consumo a essi connessi misero in secondo piano la dialisi peritoneale, perché questa determinava un guadagno significativamente inferiore. Per tale motivo la maggior parte dei bioingegneri si dedicarono al miglioramento tecnologico del sofisticato "rene artificiale". L'inizio della DP nel trattamento dell'uremia cronica risale al 1968 quando Tenckhoff propose il suo catetere per dialisi peritoneale che, pur con le successive modifiche, è quello tuttora più diffuso. Ma il vero impulso alla DP è stato dato nel 1976 da Popovich e Moncrieff che introdussero il sistema della CAPD. La semplicità e il costo estremamen- 
te limitato del materiale non riuscirono a catturare l'attenzione e l'interesse delle ditte farmaceutiche. Alcuni caparbi nefrologi italiani dei Centri di Perugia, Brescia, Mestre, Milano, Vicenza hanno creduto nella bontà della tecnica migliorandola notevolmente e stimolando i colleghi delle altre Regioni a intraprendere la stessa strada. La carenza dei posti tecnici di emodialisi e le ristrettezze economiche del Sistema sanitario hanno fatto il resto.

Soltanto negli ultimi anni, però, con l'introduzione delle apparecchiature automatiche si è notato un certo interesse da parte della maggior parte delle ditte che prima si occupavano soltanto di emodialisi.

\section{Conclusioni}

La letteratura internazionale ormai da anni ha dimostrato i vantaggi della $\mathrm{DP}$, che non sto qui ad elencare, riconoscendole pari dignità rispetto all'ED nel trattamento sostitutivo dell'uremia cronica. Come dice Socrate "noi dobbiamo attribuire il massimo valore non alla vita, ma a vivere bene" e da questo punto di vista la DP offre le maggiori chance. Le limitazioni, infatti, che maggiormente sentono i dializzati sono relative all'impiego del tempo libero, e in particolare alla possibilità di fare viaggi e vacanze. Nella nostra esperienza alcuni pazienti in DP, oltre alle vacanze tradizionali, hanno ripreso a sciare in località lontane da Centri dialisi; una paziente ha potuto trascorrere regolarmente due mesi a Filicudi in una abitazione saltuariamente sprovvista di elettricità; un'altra si reca annualmente in Giappone. Infine, con nostra grande soddisfazione, siamo riusciti a organizzare un viaggio a Cuba della durata di 45 giorni per una nostra paziente facendole pervenire con vari sotterfugi il materiale occorrente, nonostante l'embargo americano verso quello stato anche per prodotti medicali.

Vorrei infine concludere con il concetto a mio avviso più innovativo e foriero di grande rilancio della DP in Italia. DP e ED non debbono essere considerate alternative o antitetiche, ma devono essere utilizzate in maniera integrata e sequenziale. La DP offre i migliori outcomes clinici quando viene utilizzata come prima forma di terapia sostitutiva della funzione renale nei pazienti giovani. Utilizzata con tali nuove indicazioni, la DP può essere utile per programmare gli ingressi in ED là dove esistono carenze di posti ovvero costituire area di attesa di un possibile trapianto di rene.

sergio.sisca@asf.toscana.it 